\title{
Annealing-enhanced birefringence and aggregation in MEH-PPV: a spectroscopic ellipsometry study
}

\author{
Olivier P. M. Gaudin and Ifor D. W. Samuel* \\ Organic Semiconductor Centre, SUPA, School of Physics and Astronomy, University of St \\ Andrews, North Haugh, St Andrews, Fife KY16 9SS, United Kingdom \\ Samia Amriou ${ }^{1}$ and Paul L. Burn ${ }^{1,2}$ \\ ${ }^{1}$ Department of Chemistry, University of Oxford, Chemistry Research Laboratory, Mansfield \\ Road, Oxford OX1 3TA, United Kingdom \\ ${ }^{2}$ Centre for Organic Photonics \& Electronics, The University of Queensland, School of \\ Chemistry and Molecular Biosciences, Queensland, 4072, Australia
}

\begin{abstract}
We have used absorption, photoluminescence (PL) and variable angle spectroscopic ellipsometry (VASE) measurements to investigate the structural changes that take place upon high temperature annealing in spin-coated films of the prototypical conjugated polymer (CP) poly[2-(2'-ethylhexyloxy)-5-methoxy-1,4-phenylenevinylene] (MEH-PPV). Absorption and VASE measurements reveal that the birefringence of the films increases by approximately a factor of two upon heating, which indicates significant increase in the alignment of the conjugated polymer (CP) strands within the film plane. Absorption and PL spectra indicate the formation in annealed films of interchain species having lower energy transitions. But these measurements alone do not reveal the type of interchain species formed, such as excimers or aggregates. VASE measurements were used to investigate this feature and clearly reveal a new, low energy, feature with a shoulder at $650 \mathrm{~nm}$ in the dispersion relations of the extraordinary (out-of-plane) extinction and absorption coefficients of annealed films, which we assign to aggregate absorption. Thus, our work shows that VASE is a sensitive enough technique to measure aggregate absorption in CP films. In the case of the ordinary (in-plane) extinction and absorption coefficients, there is increased amplitude of the lower energy peak upon heating, owing to increased uniaxial anisotropy, along with a broadening and a longer red-tail, but the well-resolved red-shifted absorption band seen for the extraordinary absorption coefficient, is not observed. Therefore, we conclude that while in-plane and outof-plane aggregation occurs in annealed spin-coated films of MEH-PPV, aggregate absorption is only clearly observed when the aggregate electronic transition dipole is oriented preferentially in a direction perpendicular to the film plane. This conclusion is consistent with the usual observation that aggregate absorption in MEH-PPV films is not easily observed using absorption spectra alone, which are typically measured at normal incidence.
\end{abstract}




\section{Introduction}

Solution-processed organic semiconductors [1-19], such as conjugated polymers [1-7, 18, 19], dendrimers [8-10] and small molecules [11-16], are important for a wide range of electronic and optoelectronic devices such as light emitting diodes [1-3,8-10, 12-16], solar cells [4-6,11], field effect transistors [7] and lasers [17-19]. One of their advantages, compared to those processed by thermal evaporation, is that low cost deposition techniques such as spin-coating, printing [20] or spray coating [21,22] can be used to fabricate devices. However, the electronic and optical properties of solution-processed organic semiconductors, and in turn the performance of devices made from them, are strongly influenced by the way they are processed. For instance, it has been reported that the photophysical properties [2326], microstructure [27] and charge carrier transport [28] of conjugated polymer (CP) films can change significantly depending on the solvent used to cast the films. Another important factor which can drastically affect the physical properties of films of solution-processed organic semiconductors is thermal annealing [25,29-36].

Several studies have shown that thermal annealing can strongly modify not only the contact properties but also the morphology of the organic layer(s) in various types of organic devices, which include bulk heterojunction and bi-layer solar cells [6,11], field effect transistors [33], light-emitting diodes [37-40] and light-emitting electrochemical cells (LECs) [41]. In several cases, the performance of heat treated devices was found to be strongly enhanced [6,37-41], so that a high temperature treatment could in fact be used as an efficient optimization step in the device fabrication process. In view of the above changes upon heating, it is important to understand how annealing affects the physical properties of thin films made using solution-processed organic semiconductors.

MEH-PPV is a prototypical electroluminescent CP which has been widely studied but still serves today as a model material for understanding the complex behaviour CPs can have depending on how they have been synthesized and processed, and which applications they are intended to be used for. Thus, further understanding of its photophysical properties, such as molecular orientation and aggregation, remains highly relevant to research on CP films today.

Spin-coated films of MEH-PPV annealed above their glass transition temperature, $\mathrm{T}_{\mathrm{g}}$, are known to contain an increased fraction of a variety of interchain species compared to asdeposited films [25,29], yielding a weak, red shifted photoluminescence (PL) with a radiative lifetime longer than the emission lifetime from a single polymer chain [29]. The formation of such species is understandably detrimental to the performance of optoelectronic devices, such 
as OLEDs, since they will directly lead to loss of light output as well as shift in the CIE color coordinates. Therefore, being able to identify them and determine their type, such as excimer or aggregate, is important but not easy [29,42]. For aggregates, which are intermolecular ground state species, a clear red shift in the absorption spectrum has sometimes been reported [43]. However, in most cases, only a weak, red shifted feature could be observed [44,45], so that the film absorption spectrum alone is not well-suited to reveal aggregate absorption $[29,42]$. An additional difficulty that arises when using absorption is the strong impact reflectivity can have on the shape and position of absorption features. This could be particularly important for studying aggregate absorption in MEH-PPV as reflectivity can lead to a long tail on the red side of the main absorption peak, precisely in the region where aggregate absorption could be present [46]. Therefore, more sensitive techniques are needed to unambiguously reveal aggregate absorption [29].

In this paper, we use variable angle spectroscopic ellipsometry (VASE), along with absorption and steady-state photoluminescence (PL), to investigate the effect that thermal annealing above $\mathrm{T}_{\mathrm{g}}$ has on the optical properties of spin-coated films of poly[2-(2'ethylhexyloxy)-5-methoxy-1,4-phenylenevinylene] MEH-PPV. Although there has been many ellipsometric studies of CP, including several using MEH-PPV [46-48], here we investigate more specifically the impact annealing above $T_{g}$ can have on the film optical properties and whether VASE can be used as a technique to reveal aggregate absorption in semiconducting polymer films. A major advantage of VASE over absorption measurements is the ability to extract the absorption coefficient, thus removing any ambiguity that could arise from the presence of reflections affecting the absorption spectrum. A second advantage is its accuracy as it takes into account not only changes in the light intensity upon interaction with the sample, but also in the light phase through measurement of the $\Delta$ parameter [49]. A first observation is that the amplitude of the lower energy absorption peak in MEH-PPV films substantially increases in annealed films, indicating increased absorption strength for the incident light that is normal to the substrate plane. In contrast, the strength of the higher energy transition at $6 \mathrm{eV}$ remains unchanged. VASE measurements reveal that on annealing, the ordinary absorption coefficient increases, whilst the extraordinary coefficient decreases in the region of the lower energy absorption peak, but for higher energy absorption $(>5.2 \mathrm{eV})$ the absorption coefficients are unchanged. At the same time, the film birefringence increases by nearly a factor of two in annealed films. We conclude from this that there is substantial reorientation of the polymer chains upon heating, so that the observed changes to the absorption spectrum can be explained in term of re-distribution of the orientation of transition dipoles. Thus, our results reveal strong changes to the film morphology upon heating, and 
also show that molecular orientation is an important parameter to take into account when using absorption spectra on their own to compare the absorbing strength of different $\mathrm{CP}$ films.

We also find that careful analysis of the VASE data clearly reveals an additional feature at $650 \mathrm{~nm}$ in the extraordinary absorption coefficient, which we assign to aggregate absorption. In contrast, this feature is neither observed in the ordinary absorption coefficient, nor the absorption spectrum. This has two important implications; first, it indicates that VASE can be used as a technique to reveal aggregate absorption in CP films, and a second implication is that aggregate absorption in MEH-PPV films is more easily observed when the aggregate oscillator strength is polarized preferentially perpendicular to the film plane. Since absorption spectra are typically measured at normal incidence, this observation is consistent with reports that aggregate absorption is not easily observed from transmission measurements alone $[29,42]$.

\section{Experimental}

The Gilch route [50] is often used to form MEH-PPV, however, in our work we used MEH-PPV that was formed from a bis(xanthate) containing monomer. Xanthate monomers have been used to form precursor polymers, which after processing to form thin films are converted to the insoluble conjugated polymer by thermal treatment [51,52]. In this work the xanthate precursor polymer was heated in diphenylether to eliminate the xanthate groups and leave the soluble MEH-PPV. The MEH-PPV had a weight average molecular weight of $1.0 \times$ $10^{5} \mathrm{Da}$ and polydispersity of 2.5 . The solvent used for spin-coating the thin films was chlorobenzene.

The film thickness was varied by changing both the solution concentration $(5-25$ $\mathrm{mg} / \mathrm{ml})$ and the spin-coating speed $(1000-6000 \mathrm{rpm})$.. Thermal annealing was performed in a vacuum oven (low $10^{-5} \mathrm{mbar}$ ) for nine hours at a temperature of $225^{\circ} \mathrm{C}$, therefore above the $\mathrm{T}_{\mathrm{g}}$ of 196 [53] to $216{ }^{\circ} \mathrm{C}$ [23]. All the optical measurements were performed in ambient conditions, but samples were exposed to air for less than 3 hours by the completion of the measurements.

Absorption, PL and reflectance spectra were measured using films on fused silica substrates, and absorption spectra were corrected for absorbance within the substrate. PL spectra were recorded with a Fluoromax-2 spectrofluorometer from Horiba Jobin Yvon. For the VASE measurements, both fused silica and silicon substrates were used. Prior to spincoating, the substrates were cleaned by ultrasonication in acetone followed by propan-2-ol. 
VASE measurements were performed in the reflection mode over the range $190-$ $1700 \mathrm{~nm}$ using a J. A. Woollam Co., Inc. M-2000DI system. Absorption spectra were acquired both using the M-2000DI system and a Cary 300 Bio UV-Vis spectrophotometer from Varian, Inc., as a comparison. The latter was also used for measuring near normal incidence ( $7^{\circ}$ to the sample normal) absolute specular reflectance (ASR) spectra, used here only for the purpose of modelling the VASE data, as we have explained elsewhere [46]. The VASE data, as well as the absorption and ASR spectra, were modelled using the WVASE32 ${ }^{\circledR}$ analysis software. All the thicknesses were derived from the VASE measurements.

VASE data acquisition consisted of measuring, as a function of wavelength $\lambda$ and angle of incidence (AOI), $\Phi$, the standard ellipsometric parameters $\Psi$ and $\Delta$, defined as $[49,55]$ :

$\tan (\psi) e^{i \Delta}=\rho=\frac{r_{p p}}{r_{s S}}$

where $\rho$ is the ratio of the complex Fresnel reflection coefficients of the sample for the p- (in the plane of incidence) and s- (perpendicular to the plane of incidence) component of the polarised light.

In order to minimize correlation between parameters, experimental data were acquired using a procedure designed to largely over-determine the model, that is, the number of independent experimental data exceeded by far the number of fitting parameters used in the analysis. This was implemented by measuring the $\Psi$ and $\Delta$ data for a large number of MEHPPV films having significantly different thicknesses up to $178 \mathrm{~nm}$ and AOIs from $45^{\circ}$ to $75^{\circ}$. The optical parameters of as-deposited samples were determined using the VASE data from nine of these films, as we reported previously [46]. The data were processed by means of a multi-sample analysis [55,57], which combined not only the $\Psi$ and $\Delta$ data, but also the absorption spectra [57], and for some samples, the ASR spectra as well.

In addition to greatly reducing correlations between parameters $[55,56]$, this procedure also leads to a single set of optical constants averaged over the whole range of experimental data types and thicknesses. Whilst the multi-sample approach would make the data analysis inaccurate, or even impossible, if the film optical parameters depended strongly on the thickness, we have shown previously that this was not in fact the case for spin-coated films of MEH-PPV [46]. Thus, using a single set of optical parameters determined in this way, we found that for any sample with a thickness in the range $18-178 \mathrm{~nm}$, all the experimental data could be simulated accurately. 
Determination of the optical constants of annealed films was performed using a set of eight samples, with an MEH-PPV film thickness in the range $48-178 \mathrm{~nm}$, reduced to $44-$ $175 \mathrm{~nm}$ after annealing. The modeling procedure used was similar to that employed for the as-deposited films. Again, we found that a single set of optical constants could describe with good accuracy experimental data acquired for any thickness within the range. This is shown in Fig. 1(a) and (b) where a very close fit is obtained to the $\Psi$ and $\Delta$ data from both the $44 \mathrm{~nm}$ and the $175 \mathrm{~nm}$ thick films. Close fits were also obtained for any other film thickness within the range, and in the case of the transmission spectra as well (not shown). Therefore, we conclude that for annealed films as well, a single set of optical constants provides a precise description of the optical parameters for any film with a thickness in the range $44-175 \mathrm{~nm}$.

Both before and after annealing, the optical parameters were extracted using an air-CP film-substrate model, with the CP film being optically uniaxial with the optic axis perpendicular to the plane of the film $[47,48,57,58]$. No surface roughness was used. Introducing surface roughness was not found to lead to any improvement in the fit quality, which was consistent with the homogeneous and defect-free appearance of the spin-coated films. In addition, not using surface roughness removed any risk of parameter correlation between film thickness and roughness, which could be a problem for very thin films. Both the ordinary, $\varepsilon_{0}$, and extraordinary, $\varepsilon_{\mathrm{e}}$, dielectric functions (DFs) were described using a Kramers-Kronig (K-K) consistent parametric functional model of the form:

$\varepsilon_{o(e)}=\varepsilon_{o(e) 1}+i \varepsilon_{o(e) 2}=\varepsilon_{o(e) \infty}+\varepsilon_{o(e) P o l e}+\sum_{j=1}^{n} \varepsilon_{o(e), j}$,

where the first two terms on the right hand side, $\varepsilon_{o(e) \infty}$ and $\varepsilon_{o(e) P o l e}$, are real terms, whilst the third term is a summation of n complex Gaussian oscillators, noted $\varepsilon_{\mathrm{o}(\mathrm{e}), \mathrm{j}}$, which have been shown to be well-suited for describing absorption in amorphous and glassy materials $[59,60]$. In the WVASE32 ${ }^{\circledR}$ analysis software, each Gaussian term is defined as $\varepsilon_{o(e), j}=\varepsilon_{o(e) 1, j}+\mathrm{i}$ $\varepsilon_{\mathrm{o}(\mathrm{e}) 2, \mathrm{j}}$, where the imaginary part $\varepsilon_{\mathrm{o}(\mathrm{e}) 2, \mathrm{j}}$ produces a Gaussian line shape of the form:

$\varepsilon_{o(e) 2, j}=A_{o(e), j} \exp \left[-\left(\frac{E-E_{o(e), j}}{\sigma}\right)^{2}\right]-A_{o(e), j} \exp \left[-\left(\frac{E+E_{o(e), j}}{\sigma}\right)^{2}\right]$

and the real part $\varepsilon_{\mathrm{o}(\mathrm{e}) 1, \mathrm{j}}$ is obtained by $\mathrm{K}-\mathrm{K}$ transformation of the imaginary part. In Eq. (3), $\sigma$ is defined as:

$\sigma=\frac{B_{o(e), j}}{2 \sqrt{\ln 2}}$. 
$\mathrm{A}_{\mathrm{o}(\mathrm{e}), \mathrm{j}}, \mathrm{E}_{\mathrm{o}(\mathrm{e}), \mathrm{j}}$, and $\mathrm{B}_{\mathrm{o}(\mathrm{e}), \mathrm{j}}$ are variable parameters, which correspond to the amplitude, centre energy, and broadening of the Gaussian line shape, respectively, the latter being equal to the full width at half maximum. Note the positive and negative energy Gaussian terms in Eq. (3) are required for K-K consistency [59,60].

In Eq. (2), $\varepsilon_{o(e) P o l e}$ is a zero broadening oscillator known as a Pole term, which accounts for dispersion in $\varepsilon_{\mathrm{o}(\mathrm{e}) 1}$ due to absorption outside the measured spectral range, and defined as:

$\varepsilon_{o(e) \text { Pole }}=\frac{A_{o(e) \text { Pole }}}{E_{o(e) P o l e}^{2}-E^{2}}$,

whilst $\varepsilon_{o(e) \infty}$ is a real constant. Here, the variable parameters $A_{o(e) P o l e}$ and $E_{o(e) P o l e}$ are the amplitude and centre energy, respectively.

The model parameters were fitted to the experimental data using the LevenbergMarquardt algorithm, by minimizing the total weighted root Mean Squared Error (MSE) defined as:

$M S E=\sqrt{M S E_{E}^{2}+M S E_{T}^{2}+M S E_{R}^{2}}$

where $M S E_{E}^{2}$ and $M S E_{T(R)}^{2}$ represent the ellipsometric and transmission (reflection) contributions to the MSE, respectively, and are defined as:

$M S E_{E}^{2}=\frac{1}{2 N_{E}-M} \sum_{i=1}^{N_{E}}\left[\left(\frac{\psi_{i}^{M o d}-\psi_{i}^{E x p}}{\sigma_{\psi, i}^{E x p}}\right)^{2}+\left(\frac{\Delta_{i}^{M o d}-\Delta_{i}^{E x p}}{\sigma_{\Delta, i}^{E x p}}\right)^{2}\right]$

and

$M S E_{T(R)}^{2}=\frac{1}{N_{T(R)}-M} \sum_{i=1}^{N_{T(R)}}\left[\left(\frac{T(R)_{i}^{M o d}-T(R)_{i}^{E x p}}{w_{T(R)} \sigma_{T(R), i}^{E x p}}\right)^{2}\right]$ 
Here, $\Psi_{i}^{\operatorname{Mod}(E x p)}, \Delta_{i}^{\operatorname{Mod}(E x p)}$, and $T(R)_{i}^{\operatorname{Mod}(E x p)}$ are the model generated (experimental) $\Psi, \Delta$, and intensity transmission (reflection) data points, respectively, whilst $\mathrm{N}_{\mathrm{E}}$ and $\mathrm{N}_{\mathrm{T}(\mathrm{R})}$ are the number of experimental $(\Psi, \Delta)$ pairs and intensity transmission (reflection) experimental data points, respectively. $\mathrm{M}$ is the number of variable model parameters; $\sigma_{\Psi, i}^{E x p}, \sigma_{\Delta, i}^{E x p}$, and $\sigma_{T(R), i}^{E x p}$ are the standard deviations on the $\Psi, \Delta$, and intensity transmission (reflection) experimental data points, respectively. Finally, $\mathrm{W}_{\mathrm{T}(\mathrm{R})}$ is the weighting ratio for the transmission (reflection) data. As seen from Eq. (8), decreasing $\mathrm{w}_{\mathrm{T}}$ leads to a larger contribution of intensity transmission data to the total MSE defined in Eq. (6). Here, a value of $\mathrm{w}_{\mathrm{T}(\mathrm{R})}=0.1$ was found to be a good choice in most cases. The fitting procedure used led to MSE values of 6.434 and 6.388 for the sets of, respectively, as-deposited and annealed films. Given the large number of samples fitted together, these MSE values are very low, indicating that the uniaxial model used was sufficient to yield a precise modelling of the experimental data. In addition, the experimental data were insensitive to rotation of the samples around an axis normal to the substrate plane. Therefore, using a biaxial model was not justified in this study. The values of the variable model parameters obtained following the fitting procedure both for as-deposited and annealed samples are given in the Supporting Information.

\section{Results}

\subsection{Enhanced birefringence}

The effect of annealing on the film absorption spectra is shown in Fig. 2 for an MEHPPV sample with a thickness of $178 \mathrm{~nm}$ before annealing, reduced to $175 \mathrm{~nm}$ following annealing. However, note that similar changes could also be observed for thinner films. Fig. 2 shows that before annealing the absorption spectrum is characterized by an onset at $\sim 590$ $\mathrm{nm}(2.1 \mathrm{eV})$ and four absorption bands that we label I - IV from the longer to the shorter wavelengths, according to previous reports [61,62]. Note that the actual onset value depends to some extent on the thickness of the film due to reflectivity affecting strongly the initial rise of the lower energy absorption peak [46].

Following annealing, peaks III and IV remain unchanged, but strong differences can be observed in the case of peak I and, to a lesser extent, peak II. First, peak I is broader than in the as-deposited film, particularly on the red side, which leads to a $20 \mathrm{~nm}$ red shift of the absorption onset, now observed at $\sim 610 \mathrm{~nm}$ in the annealed film. Peak I also shows increased amplitude in the case of the annealed film. 
Fig. 3 shows the dispersion relations of the refractive index for the spin-coated MEHPPV films both before and after annealing, as derived from VASE measurements. The dispersion relations of the extinction and absorption coefficients are shown in Fig. 4(a) and (b), respectively. Both before and after annealing, the ordinary (in-plane) dispersion relations differ significantly from the extraordinary (out-of-plane) dispersion relations, therefore indicating strong uniaxial optical anisotropy for both types of samples. However, the optical parameters of the annealed samples differ significantly from those of the as-deposited films.

In the as-deposited films, both the shape of the dispersion relations and the optical constant values are in good agreement with published data [47,57], providing differences in molecular weights are considered. Here, the ordinary, $\mathrm{n}_{\mathrm{o}}$, and extraordinary, $\mathrm{n}_{\mathrm{e}}$, indices of refraction have, respectively, values of 1.67 and 1.59 at $1000 \mathrm{~nm}$, which yields a birefringence $\Delta \mathrm{n}$, defined as $\Delta \mathrm{n}=\mathrm{n}_{\mathrm{o}}-\mathrm{n}_{\mathrm{e}}$, of 0.08 at a wavelength of $1000 \mathrm{~nm}$. At the same wavelength, Tammer et al. [47] reported a $\Delta \mathrm{n}$ value of 0.14 , therefore significantly higher than here. However, this was for an MEH-PPV with an $\mathrm{M}_{\mathrm{w}}$ of $1.6 \times 10^{6} \mathrm{Da}$ [57], therefore much higher than here. Koynov et al. [57] have shown that the birefringence in spin-coated films of MEH-PPV increased with the molecular weight. Thus, from the data shown in Fig. 3 of Ref. [57] we extrapolate $n_{o}=1.67, n_{e}=1.59$ and $\Delta n=0.08$ for an MEH-PPV with an $M_{w}$ of $10^{5} \mathrm{Da}$ and at a wavelength of $1064 \mathrm{~nm}$. From the data analysis performed here, we find at $1064 \mathrm{~nm}$ essentially the same values as those given above at $1000 \mathrm{~nm}$, that is values identical to those extrapolated from Ref. [57] for the same $M_{w}$ as used here. Therefore, the differences we observe between our data and those of Tammer et al. [47] can be fully explained in term of $\mathrm{M}_{\mathrm{w}}$ differences.

The level of uniaxial anisotropy in the as-deposited films of MEH-PPV can also be inferred from the dispersion relations of the extinction and absorption coefficients in the absorbing region. Thus, as seen in Fig. 4(a) and (b), in the spectral region of the four absorption peaks, the ordinary extinction (absorption) coefficient, $\mathrm{k}_{\mathrm{o}}\left(\alpha_{\mathrm{o}}\right)$, is higher than the extraordinary coefficient, $\mathrm{k}_{\mathrm{e}}\left(\alpha_{\mathrm{e}}\right)$. We also note that the extraordinary coefficient versus wavelength curves show approximately the same features as those corresponding to the ordinary coefficients. This indicates that essentially the same optical transitions are responsible for both the in-plane and out-of-plane dispersion relations. As the transition dipoles for the two lowest energy electronic transitions in PPV derivatives have been shown to be polarised essentially along the long molecular axis [63-65], the usual interpretation of such dispersion relations is that the CP chains segments are preferentially aligned in the plane of the film, that is parallel to the substrate surface $[47,48,57,58,66,67]$. 
Quantitative information about molecular orientation can be obtained from the measurement of the ratio $\mathrm{k}_{\mathrm{e}} / \mathrm{k}_{\mathrm{o}}$ and the angular distribution $\mathrm{d}_{\pi \theta}$, defined as [58]:

$$
d_{\pi \theta}=\frac{1}{1+\frac{k_{e}}{2 k_{o}}},
$$

where $\theta$ refers to the polar angle between the molecular axis and the normal to the film. For a uniform molecular distribution, $\mathrm{d}_{\pi \theta}=2 / 3$ is expected, whilst a value close to 1 should be obtained in the case of a strongly uniaxial film. The ratio $\mathrm{k}_{\mathrm{e}} / \mathrm{k}_{\mathrm{o}}$, however, and in turn the angular distribution $\mathrm{d}_{\pi \theta}$, are fairly strong functions of the wavelength, so that in practice, their determination is often averaged over several relevant wavelengths. In the case of MEH-PPV films, Tammer et al. [47] have used the range $375-565 \mathrm{~nm}(2.2-3.3 \mathrm{eV})$, that is the wavelength range of peak I for which electronic transitions involving the delocalised $\pi$ states are observed [64,65,67,68], whilst McBranch et al. [58] used three specific wavelengths at 457.9, 488 and $514.5 \mathrm{~nm}$. Following the procedure used by Tammer et al. [47], we find, for the as-deposited films, $\mathrm{k}_{\mathrm{e}} / \mathrm{k}_{\mathrm{o}}=0.51$ and $\mathrm{d}_{\pi \theta}=0.80$, whilst the method used by McBranch et al. [58] yields $\mathrm{k}_{\mathrm{e}} / \mathrm{k}_{\mathrm{o}}=0.49$ and $\mathrm{d}_{\pi \theta}=0.80$. If all the molecules pointed in the same direction, this would lead to a polar angle $\theta$ of $64^{\circ}$ using either of the two methods. The values found here for $\mathrm{d}_{\pi \theta}$ and $\theta$ are close to those derived by McBranch et al. [58], who found $\mathrm{d}_{\pi \theta}=0.84$ and $\theta=67^{\circ}$ for an MEH-PPV having the same molecular weight as the one used here. As expected for a higher molecular weight MEH-PPV [57], Tammer et al. reported a higher $\mathrm{d}_{\pi \theta}$ value of 0.91 . Therefore, we conclude that the dispersion relations found here for asdeposited films of MEH-PPV are fully consistent with previous reports [47,57].

In the annealed films, we observe that, for wavelengths longer than $550 \mathrm{~nm}$, that is in the transparent region and over the initial rise of peak $I, n_{o}$ and $n_{e}$ have respectively increased and decreased, therefore leading to an increase in $\Delta \mathrm{n}$. Thus, at a wavelength of $1000 \mathrm{~nm}$, we now find $n_{o}=1.70$ and $n_{e}=1.55$, leading to $\Delta n=0.15$. The birefringence in the annealed films is therefore nearly twice as high as in the as-deposited films. Inspection of the dispersion relations of the extinction and absorption coefficients of annealed films reveals that in the absorbing region, $\mathrm{k}_{\mathrm{o}}\left(\alpha_{\mathrm{o}}\right)$ and $\mathrm{k}_{\mathrm{e}}\left(\alpha_{\mathrm{e}}\right)$ have respectively increased and decreased, so that, using the same procedure as in the case of as-deposited films, we now find $\mathrm{k}_{\mathrm{e}} / \mathrm{k}_{\mathrm{o}}=0.25$ and $\mathrm{d}_{\pi \theta}=0.89$, from which we derive $\theta=71^{\circ}$. We conclude from these observations that the level of uniaxial anisotropy has significantly increased upon annealing, which indicates that after annealing the $\mathrm{CP}$ chain segments tend to be more aligned in the film plane. 


\subsection{Enhanced aggregation}

Fig. 5 shows the dispersion relations of the absorption coefficient, both before and after annealing, over the spectral region of the initial rise of peak I. In the as-deposited films, we observe that $\alpha_{e}$ is slightly higher than $\alpha_{o}$ over a short wavelength range of about $80 \mathrm{~nm}$ from $\sim 580-660 \mathrm{~nm}$. In contrast, in the annealed film, this region has extended over approximately $200 \mathrm{~nm}$, from 600 to $800 \mathrm{~nm}$. The relative amplitude of $\alpha_{\mathrm{e}}$ compared to $\alpha_{\mathrm{o}}$ has also increased drastically, with $\alpha_{\mathrm{e}}$ now displaying a distinct shoulder at $\sim 650 \mathrm{~nm}$. It has sometimes been pointed out that higher uncertainties exist in the determination of the extraordinary, as opposed to the ordinary, optical constants derived from reflection VASE measurements [47], or that strong parameter correlations may lead to unreliable optical constants [66]. Therefore, it may be argued that the observed differences between $n_{o}$ and $n_{e}$ over the initial rise of peak I are only due to model inaccuracies arising from a lack of sensitivity of the experimental data to small changes in the out-of-plane optical constants. Although we observed that, over the spectral range of peak IV, the details of the extraordinary dispersion relations could depend to some extent on the modelling procedure used, this was not the case at longer wavelengths where the shoulder in $\alpha_{e}$ could always be observed, as we now show more quantitatively using Figs. 6 - 8 .

Fig. 6(a) and (b) show the imaginary part of the ordinary dielectric function, $\varepsilon_{02}$, derived from the VASE data analysis, along with the set of Gaussian oscillator functions used to describe it according to Eq. (2) - (5), respectively before and after annealing the films. It can be seen in Fig. 6(b) that the main effect of annealing on $\varepsilon_{02}$ is an increase in the amplitude of the three higher energy oscillators contributing to peak I, along with a slight red-shift of all the four oscillators, which accounts for the broadening of the lower energy absorption peak in annealed films, as observed in Fig. 2. Note also in Fig. 6(b) that a low amplitude oscillator has been added to peak II, in order to account for the slightly sharper shape of this absorption peak in the annealed films. However, strong redistribution upon annealing of the oscillator centre energies in the dispersion relation of $\varepsilon_{02}$ is not observed.

Fig. 7(a) and (b) show equivalent data in the case of the imaginary part of the extraordinary dielectric function, $\varepsilon_{\mathrm{e} 2}$. Here, it can be seen that the main effect of annealing is not only a redistribution of the oscillator strengths in peak I upon annealing, but also a strong redistribution of the oscillator centre energies within this peak. This is particularly obvious in 
the case of the lower energy Gaussian oscillator whose centre energy is observed to shift from $530 \mathrm{~nm}(2.33 \mathrm{eV})$ in the as-deposited film to $620 \mathrm{~nm}(2.00 \mathrm{eV})$ in the annealed film, leading to the shoulder observed at $650 \mathrm{~nm}$ in the dispersion relation of $\alpha_{\mathrm{e}}$ for the annealed films in Fig. 5.

Fig. 8(a) shows experimental and simulated $\Psi$ data for an AOI of $70^{\circ}$ in the case of a $124 \mathrm{~nm}$-thick annealed film. Here the simulated $\Psi$ data have been obtained either using the full set of seven Gaussian oscillators shown in Fig. 7(b) or after setting the amplitude of the lower energy Gaussian oscillator to zero but leaving unchanged the six other Gaussian functions. Fig. 8(b) shows equivalent data in the case of the $\Delta$ parameter. It can be seen that complete overlap between the experimental and simulated $\Psi$ and $\Delta$ data can only be obtained when the lower energy oscillator is present in the dispersion relation of $\varepsilon_{\mathrm{e} 2}$. In order to assess the closeness between simulated and experimental data in a meaningful way, that is independent of the number $\mathrm{M}$ of adjustable parameters used, we employ a modified version of the MSE defined in Eq. (6) - (8), noted $\mathrm{MSE}_{0}$, obtained by setting $\mathrm{M}=0$ in Eqs. (7) and (8). Such a calculation in the case of the $\Psi$ and $\Delta$ data shown in Fig. 8, that is over the spectral range of $500-900 \mathrm{~nm}$ and for an $\mathrm{AOI}$ of $70^{\circ}$, leads to $\mathrm{MSE}_{0}=5.553$ when the full set of seven Gaussian oscillator functions is used to simulate the $\Psi$ and $\Delta$ data. In contrast, $\mathrm{MSE}_{0}$ increases to 19.51 after the amplitude of the lower energy Gaussian oscillator has been set to zero, therefore indicating quantitatively that the fit quality has drastically deteriorated in such a case. Note that the same behaviour was also observed for all the eight annealed samples used in the analysis.

Therefore, we conclude from this that a low amplitude Gaussian oscillator, with a centre energy at $620 \mathrm{~nm}$, is indeed required in the extraordinary dispersion relation of the dielectric function of annealed films in order to precisely model the VASE data in the region of the initial rise of the lowest energy electronic transition. This shows that the shoulder at $650 \mathrm{~nm}$ observed in the dispersion relation of $\alpha_{\mathrm{e}}$ for the annealed film in Fig. 5 is not an artefact but can be assigned to structural differences between annealed and as-deposited MEH-PPV films. In the discussion below, we show that these changes can be assigned to enhanced aggregation in the annealed films.

\section{Discussion}

The photophysical properties of spin-coated films of MEH-PPV annealed above $\mathrm{T}_{\mathrm{g}}$ have previously been studied [23-25,29,70]. It was found that such films contained an 
increased fraction of interchain interactions compared to as-cast films and could form interchain species such as excimers, aggregates, polaron pairs or exciplexes. These interchain species were characterized by the presence of a weak, red-shifted, feature in the PL spectrum, with a radiative lifetime much longer than the lifetime of intrachain excitons. The absorption spectra of annealed films were also broader and red-shifted compared to those of as-deposited films, a feature that was assigned to the existence of ground-state interchain species, that is, aggregates, along with, possibly, increased effective conjugation length [29].

Here, we also observe similar changes to the absorption spectrum upon annealing, as can be clearly seen in Fig. 2. Also shown in Fig. 2 are the PL spectra of the film, both before and after annealing. In the as-deposited film, we observe a main peak at $585 \mathrm{~nm}$ along with a shoulder at $623 \mathrm{~nm}$, as expected for non-annealed MEH-PPV films [23]. In contrast, the PL spectrum of the annealed film is broader and red-shifted. Its integrated emission intensity was also reduced to $40 \%$ of that of the un-annealed sample. The broadening of the absorption spectrum, the increase in the average conjugation length, and the changes to the PL spectra indicate that the thermal treatment used here was performed above $T_{g}$ and that it led to formation of an increased fraction of interchain species in the annealed films [29]. However, on the basis of the PL and absorption spectra alone, the exact nature of the interchain species remains unclear. Therefore, we turn our attention to the VASE results. The new and wellresolved low energy feature that we observe at $\sim 650 \mathrm{~nm}$ in the dispersion relation of $\alpha_{\mathrm{e}}$ for annealed films strongly suggests that new electronic species, having lower energy transitions, that is aggregates, have formed.

This assignment is supported by a previous study. Using a near-field scanning electron microscopy (NSOM)-based solvatochromism method, Schaller et al. have shown that the majority of interchain species in the near-surface region of annealed MEH-PPV films were delocalised with little charge separation [29]. In order to establish whether the films contained excimers or aggregates a combination of third harmonic generation (THG) and NSOM was used, the former being sensitive enough to detect aggregate absorption. THGNSOM spectra of annealed spin-coated films of soluble MEH-PPV revealed a large number of aggregation domains characterized by a distinct absorption feature over the range $620-$ $670 \mathrm{~nm}$, with a peak at $650 \mathrm{~nm}$, as is observed here in the dispersion relation of $\alpha_{\mathrm{e}}$. Although in principle such a low energy feature could result merely from increased conjugation length in some regions of the samples, such an assignment was thought to be unlikely because the electron delocalisation along the CP backbone is known to saturate before intrachain excitons can absorb at such long wavelengths [29]. In as-deposited films, the relative increase in $\alpha_{\mathrm{e}}$ compared to $\alpha_{\mathrm{o}}$ that is observed over a small wavelength range at low energies could then also 
be accounted for by the presence of aggregates, but in much lower concentration. In fact, this would also be in close agreement with the THG-NSOM measurements reported in Ref. [29], since aggregation domains could also be observed occasionally in pristine films.

This assignment to aggregates is also supported by the reduction in thickness observed for all eight annealed samples by 1 to $4 \mathrm{~nm}$ depending on the sample. This result is in agreement with previous neutron reflectivity measurements [53] and suggests an increased film density in annealed films, which would then likely promote aggregate formation due to increased CP chain packing. The previous observations have a number of important implications. First, our measurements show that VASE can be used to measure aggregate absorption in CP films, which further extends the amount of information that can be accessed using VASE. Secondly, since the low energy absorption feature is only observed in the case of the extraordinary absorption coefficient, we conclude that it originates from aggregates having an electronic transition polarized preferentially perpendicular to the film plane. It has been reported that aggregate absorption in MEH-PPV films is difficult to observe from transmission measurements alone, for which a significant background level compared to the aggregate absorption strength can be present [29]. In addition, we note here that since absorption spectra are typically measured at normal incidence the configuration of the experiment alone may not in fact be well suited to measure an electronic transition whose transition dipole moment would be polarized mainly perpendicular to the substrate plane.

The above assignment raises a question of why aggregates should be preferentially polarised out of, rather than in, the film plane. Aggregate formation has been extensively studied in the case of the cyanine and isocyanine classes of dyes [71-74]. A number of studies have also focused on conjugated oligomers, including poly( $p$-phenylene vinylene) (PPV) oligomers [73-79], or polymers [43-45,82-89], such as PPV derivatives [44,83,89], polydiacetylene (PDA) [88] and poly(3-n-hexylthiophene) (P3HT) [86-88]. The classical model often used to explain the geometry and the photophysical properties of aggregates in these systems, particularly in the case of the cyanine dyes [71-74] and conjugated oligomers [75-81], is the molecular exciton model, which applies to strongly interacting chromophores [90]. The model predicts the formation of two types of aggregates, known as H- and Jaggregates, depending on the relative arrangement of the interacting transition dipoles. In the case of parallel transition dipoles, i.e., plane-to-plane stacking, only the in-phase dipole arrangement, as depicted in Fig. 9 (a), should lead to an allowed transition from the ground state to the exciton state and the singlet-singlet transition in the aggregate should be blueshifted compared to the transition observed in non-interacting chromophores. This is the case of H-aggregates. In contrast, if the transition dipoles in the aggregate are in-line, i.e., head-to- 
tail stacking, then only the in-phase dipole arrangement, as depicted in Fig. 9 (b), is predicted to lead to an allowed transition from the ground state to the exciton state but the singletsinglet transition in the aggregate is expected to be red-shifted with respect to the transition in the isolated chromophore, leading to the J-type aggregates. If we call $\beta$ the slippage angle, that is the angle formed between the polarisation axis and the line of molecule centres, then the $\mathrm{H}$ - and J-aggregates would correspond to $\beta$ values of $90^{\circ}$ and 0 , respectively. Note that for $\beta$ values in between, the aggregate-allowed exciton state is expected to vary continuously between a blue- and a red-shifted electronic transition, so that the amount and direction of the shift that is expected in the absorption and PL spectra upon aggregate formation, with respect to non-interacting chromophores, is in fact closely related to the aggregate geometry. This third type of aggregate is illustrated in Fig. 9 (c).

The classical model outlined above has often been used to describe aggregates involving a limited number of chromophores, for instance dimers, and having a well-defined conjugation length, as is the case for oligomers [75-81]. In comparison, the situation that prevails in CP films is expected to be more complex due to both disorder and the amorphous nature of the films [83]. However, the predictions of the molecular exciton theory are still relevant, as they indicate that aggregate formation is not expected to lead necessarily to a well-defined, low energy, feature in the film absorption spectrum, but rather to a much wider range of possible spectral changes depending on the details of the molecular packing.

Liu et al. [37] studied the effect of thermal annealing above $T_{g}$ on the performance of MEH-PPV-based polymer light emitting diodes using reflective absorption Fourier transform infrared spectroscopy (RA-FTIR). They found that re-orientation of the MEH-PPV molecules occurred upon annealing, with both the phenyl rings and the vinyl double bonds becoming more in-plane, that is more parallel to the substrate. This picture is consistent with both the increased uniaxial anisotropy that we observe here in annealed films, and the increased aggregation since parallel orientation of the aromatic rings is expected to promote $\pi-\pi$ interaction, thus encouraging aggregate formation [23,35,36,72,83]. Since the electronic transition dipole related to peak I is considered as being polarized essentially along the MEHPPV molecule main axis [63-65], we conclude that such a parallel arrangement is well-suited to the formation of $\mathrm{H}$ - or J-type aggregates, or a configuration in between if there were slippage of neighbouring $\mathrm{CP}$ strands. In addition, considering that some $\mathrm{CP}$ segments remain oriented preferentially out of the substrate plane as evidenced by the ellipsometry measurements which indicate a reduced but non-zero extraordinary absorption coefficient upon annealing, it can be envisaged that aggregates may form not only between CP strands 
aligned preferentially within the film plane but also between MEH-PPV molecules with a tendency to be perpendicular to it, or a combination of both.

Therefore, based on the classical theory developed by Kasha [90], and assuming dipoles oriented parallel one another within the aggregates (an aggregate configuration referred to in Kasha's work as parallel transition dipole orientation [90]), a possible explanation of the results we observe is that the slippage angle is not the same for aggregates that form between CP strands that are aligned in the film plane and those that tend to be out of the film plane. This would imply there exists a distribution of aggregates having a more or less pronounced H- or J-type character depending on the specific orientation of the individual molecules in the aggregate. More specifically, aggregates whose individual dipoles tend to be normal to the substrate plane would have more J-type character, hence leading to the redshifted feature observed in $\alpha_{e}$ of annealed films. In contrast, aggregates that form from CP segments that are more aligned within the film plane would be more of the H-type. Such a distribution of aggregates would be consistent with a recent study by Chakraborty and Rothberg [91] which suggested the presence in MEH-PPV films of a very inhomogeneous distribution of emissive sites leading to both $\mathrm{H}$ - and J-like regions. However, Chakraborty and Rothberg [91] assigned the J-like character they observed to intra-chain coupling. Further, it is unlikely that strong aggregation between CP strands that are significantly misaligned within the film plane, as would be required for observing J-type aggregates with distinct out-of-plane absorption (and non-observable in-plane absorption), actually occurs. Therefore, alternative explanations should be considered. Spano and co-workers recently introduced the $\mathrm{HJ}$-aggregate model, which predicts that conjugated polymer aggregates of cofacially arranged polymer chains have a photophysical response determined by a competition between intrachain coupling, which favours J-aggregate-like behaviour, and interchain coupling, which promotes H-aggregate-like characteristics [88]. For example, it was shown that in spin-coated films of regioregular P3HT, face-to-face stacking of the $\mathrm{CP}$ chains in aggregates led to $\mathrm{H}$-aggregate behaviour due to interchain interactions being stronger than intrachain coupling [86,88]. In contrast, in aggregates of P3HT nanofibers [87], red phase PDA [88], or red phase MEH-PPV chains [89], a J-like behaviour could be observed owing to increased intrachain order, which led to strong intrachain coupling. Although we cannot rule out from the measurements made here that intrachain coupling could lead to an extra, low energy, feature in $\alpha_{\mathrm{e}}$ of the type we observe here, we consider it to be unlikely because it would require substantial elongation of the polymer chains within the films along with a level of ordering which seems difficult to obtain using conventional deposition techniques such as spin-coating $[83,87]$, especially for $\mathrm{CP}$ segments that tend to be out-of plane. 
In fact, an alternative and more straightforward interpretation of the VASE data may be provided if it is now assumed the individual dipoles forming the aggregates are not parallel one another, but at a certain angle $\delta$ (an aggregate configuration referred to in Kasha's work [90] as oblique transition dipoles, see Fig. 9). In such a case, it is required to distinguish between in-phase and out-of-phase arrangement of transition dipoles, as both types of arrangement can lead to allowed transitions [90]. If it is assumed the aggregate is a dimer and forms between a CP strand that is aligned within the film plane and another one that is at a small angle to it, the classical theory now predicts that the dipole arrangement depicted in Fig. 9 (d), referred to by Kasha as out-of-phase dipoles, will lead to an increased excited state energy, that is a blue shift. In contrast, the dipole arrangement shown in Fig. 9 (e), referred to by Kasha as in-phase dipoles, will lead to an excited state energy decrease, that is a red shift. Further, the out-of-phase and in-phase oscillator strengths are predicted to be polarized perpendicularly to one another, so that the main absorption features related to these aggregates should be, respectively, in- and out of-the substrate plane. In such a case, the red-shifted feature that is observed in the dispersion relation of $\alpha_{e}$ of annealed films could be assigned to in-phase arrangement of oblique transition dipoles, that is a disordered aggregate. In contrast, the out-of-phase dipole arrangement should lead to a blue-shift. However, due to the strong in-plane absorption from non-aggregated chains, it may not in fact easily be observed in the dispersion relation of $\alpha_{0}$. Structural relaxation in the aggregate could also contribute to lower its energy. As there exists within the pristine MEH-PPV film transition dipoles that are both aligned within the film plane and at a certain angle to it, it is in fact quite likely that when the film becomes denser upon annealing above $T_{g}$ and that the MEH-PPV molecules further align in the film plane, some disordered aggregates of the type described above will form. It is therefore believed the most likely explanation of the results we observed is due to the formation of disordered aggregates, most likely dimers, between a CP strand that is aligned within the film plane and one that is at a small angle to it.

\section{Conclusions}

We have used a combination of absorption, VASE, and PL measurements to investigate the physical changes that take place when a conjugated polymer film is annealed. Spin-coated films of MEH-PPV were annealed above $\mathrm{T}_{\mathrm{g}}$, and absorption measurements indicated there is a strong increase in the amplitude of the lowest energy absorption peak after annealing. Whilst this may appear as being due to increased absorption strength in 
annealed films, VASE measurements showed this is not in fact entirely the case. They revealed that annealed films of MEH-PPV have a birefringence approximately twice that of as-deposited films, which indicates strong molecular re-orientation upon heating, leading to increased film uniaxial anisotropy. Thus, in annealed films of MEH-PPV, the CP chains tend to be more aligned in the plane of the film, leading to increased absorption strength for incident light normal to the substrate plane, as is the case when measuring an absorption spectrum, but not parallel to it. Absorption and PL measurements showed that annealed films contain an increased fraction of interchain species compared to as-deposited films. However, the type of species formed could not be determined from such measurements alone as no clear feature from a ground state species could be observed.

In contrast, VASE results revealed the existence of a new, low energy, feature in the dispersion relation of the extraordinary absorption (and extinction) coefficient of annealed films over the spectral range $600-800 \mathrm{~nm}$, which we assigned to lower energy ground-state electronic species, that is, aggregates. For such films, only a long red tail in the dispersion relation of $\alpha_{0}$ could be observed. Using the classical molecular exciton theory, we showed that a possible explanation for these differences at longer wavelengths between ordinary and extraordinary absorption coefficients of annealed films could be variations in the aggregate geometry as a function of the CP chain orientation within the film. Thus, for aggregates formed between $\mathrm{CP}$ strands that tend to be perpendicular to the film plane, a strong enough slippage between individual transition dipoles could in principle provide a well-resolved, redshifted, feature in the absorption spectrum, as can be the case for J-type aggregates. However, strong aggregation was thought to be unlikely to happen between CP segments whose orientation is strongly out of the plane. Increased intrachain exciton coupling could in principle also lead to the observed J-aggregate behaviour, as inferred by the newly published HJ-aggregate model [85]. However, this would imply substantial elongation of out-of-plane polymer chains upon annealing, which is not expected due to the strongly increased uniaxial anisotropy. Instead, we assigned the observed low energy feature at $650 \mathrm{~nm}$ in $\alpha_{\mathrm{e}}$ to disordered aggregates, most likely dimers, that would form in between oblique, in-phase, transition dipoles

Therefore, the work presented here indicates that VASE is useful to detect aggregate absorption in CP films, something that is difficult to detect using absorption spectra alone. Our results provide further evidence for the presence and importance of aggregates in conjugated polymer films. In addition, they show the strong influence of processing on the structural and photophysical properties of conjugated polymer films. 


\section{Acknowledgements}

We are grateful to the EPSRC of the UK for financial support (grant GR/S62628/01). I.D.W.S. also acknowledges support from a Royal Society Wolfson Research Merit Award. The research data supporting this publication can be found at https://doi.org/10.17630/0a53cbf3-5772-46b3-bfd3-a04bbb2b0390 


\section{Figure captions:}

\section{Figure 1:}

Experimental (symbols) and simulated (solid lines) ellipsometric $\Psi$ (a) and $\Delta$ (b) data measured at an AOI of $65^{\circ}$ to the sample normal for two annealed films of MEH-PPV on fused silica substrates with thicknesses of 44 and $175 \mathrm{~nm}$.

\section{Figure 2:}

Absorption and PL spectra measured for the 178 nm-thick film of MEH-PPV shown before and after annealing. The film thickness was reduced to $175 \mathrm{~nm}$ after annealing.

\section{Figure 3:}

Dispersion relations of the ordinary, $\mathrm{n}_{\mathrm{o}}$, and extraordinary, $\mathrm{n}_{\mathrm{e}}$, refractive indices of the MEHPPV films before (closed symbols) and after (open symbols) annealing.

\section{Figure 4:}

Dispersion relations of the ordinary and extraordinary extinction, $\mathrm{k}_{\mathrm{o}}$ and $\mathrm{k}_{\mathrm{e}}$, (a) and absorption, $\alpha_{o}$ and $\alpha_{e}$, (b) coefficients of the MEH-PPV films before (closed symbols) and after (open symbols) annealing.

\section{Figure 5:}

Same data as in Fig. 4(b) but over the wavelength range $550-900 \mathrm{~nm}$.

\section{Figure 6:}

Dispersion relations of the imaginary part of the ordinary dielectric function, $\varepsilon_{02}$, of the MEH-PPV films before (a) and after (b) annealing. The set of Gaussian oscillator functions used to model $\varepsilon_{02}$ is also shown.

\section{Figure 7:}

Dispersion relations of the imaginary part of the extraordinary dielectric function, $\varepsilon_{\mathrm{e} 2}$, of the MEH-PPV films before (a) and after (b) annealing. The set of Gaussian oscillator functions used to model $\varepsilon_{\mathrm{e} 2}$ is also shown. 


\section{Figure 8:}

Experimental (symbols) and simulated ellipsometric $\Psi$ (a) and $\Delta$ (b) data measured at an AOI of $70^{\circ}$ to the sample normal for a $124 \mathrm{~nm}$-thick annealed film of MEH-PPV on fused silica substrate over the range $500-900 \mathrm{~nm}$. The simulated curves have been generated either using the full set of seven Gaussian oscillators shown in Fig. 7(b) (solid line) or after removing the lower energy Gaussian oscillator from the set (dot dashed line).

\section{Figure 9:}

Schematic showing different types of aggregates (dimers) depending on the specific dipole arrangement. In the case of parallel transition dipoles, the type of aggregate varies depending on the $\beta$ angle between the transition dipole direction and the line of molecular centres from an H-aggregate (a) to a J-aggregate (b) or a configuration in-between (c). Note that in (a) (c), all the dipoles are in-phase. In the case of oblique dipoles, both out-of-phase (d) and inphase (e) dipoles need to be considered as both types of dipole arrangements are expected to lead to an allowed transition from the ground state the exciton state. In (d) and (e), $\delta$ is the angle between the two transition dipole directions in the dimer. 
Figure 1

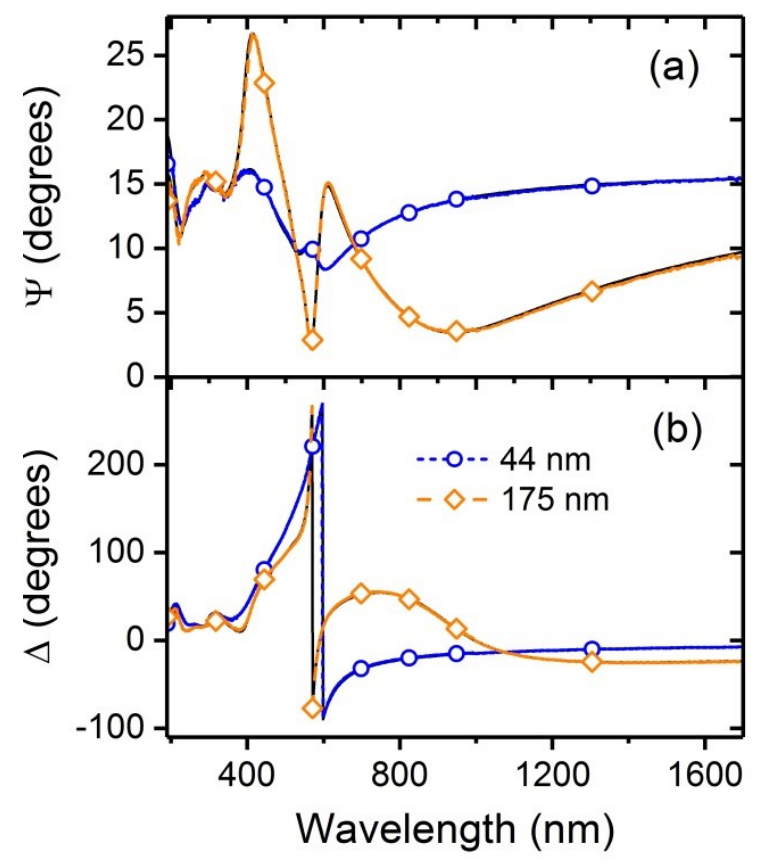


Figure 2

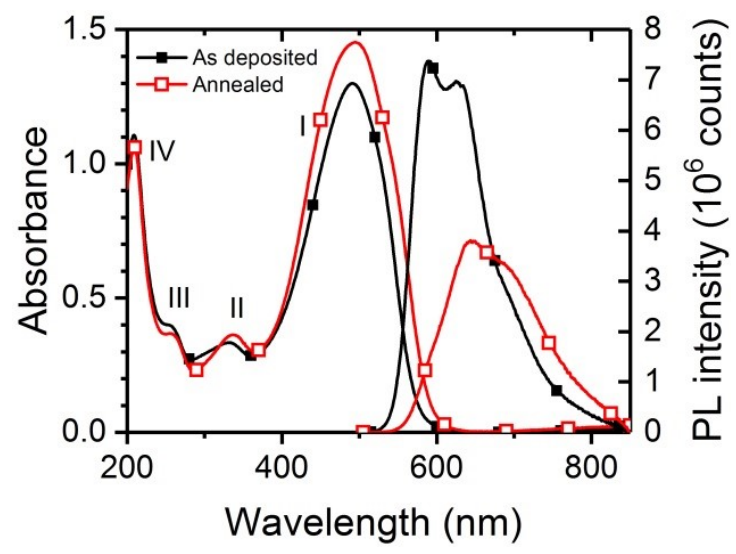


Figure 3

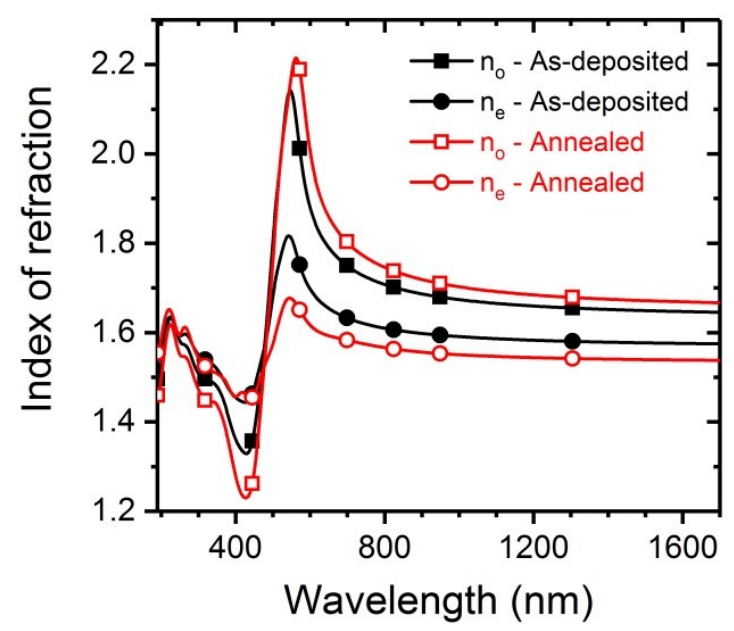


Figure 4

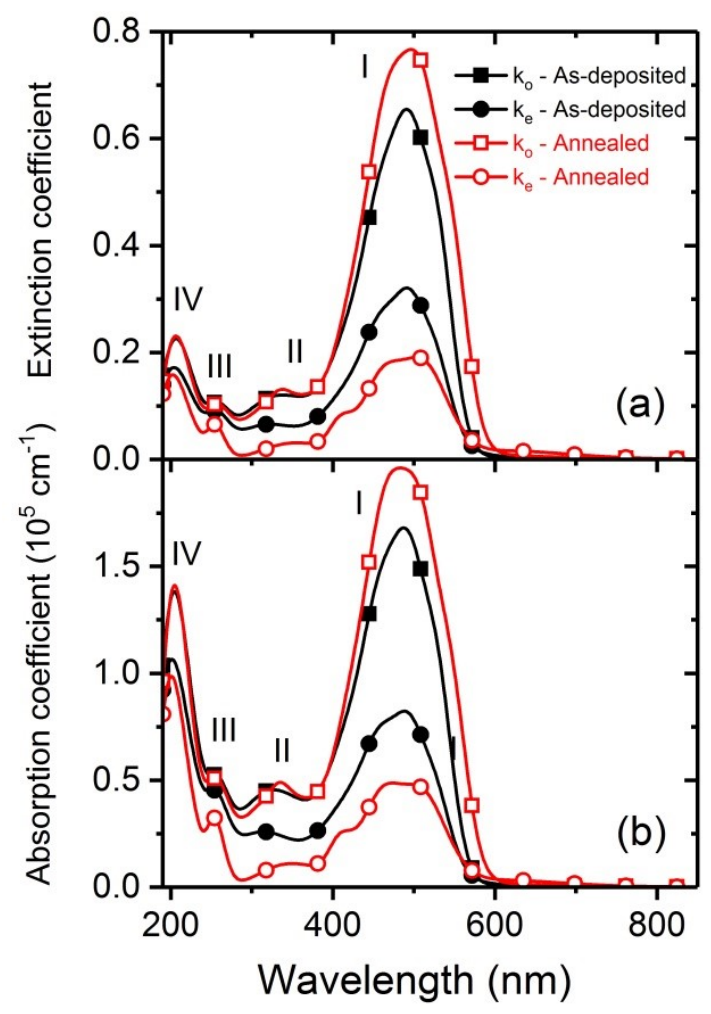


Figure 5

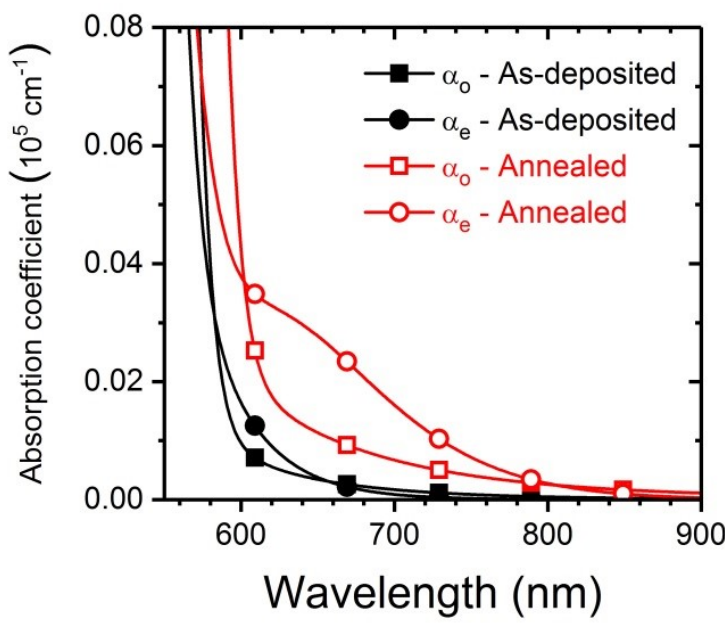


Figure 6

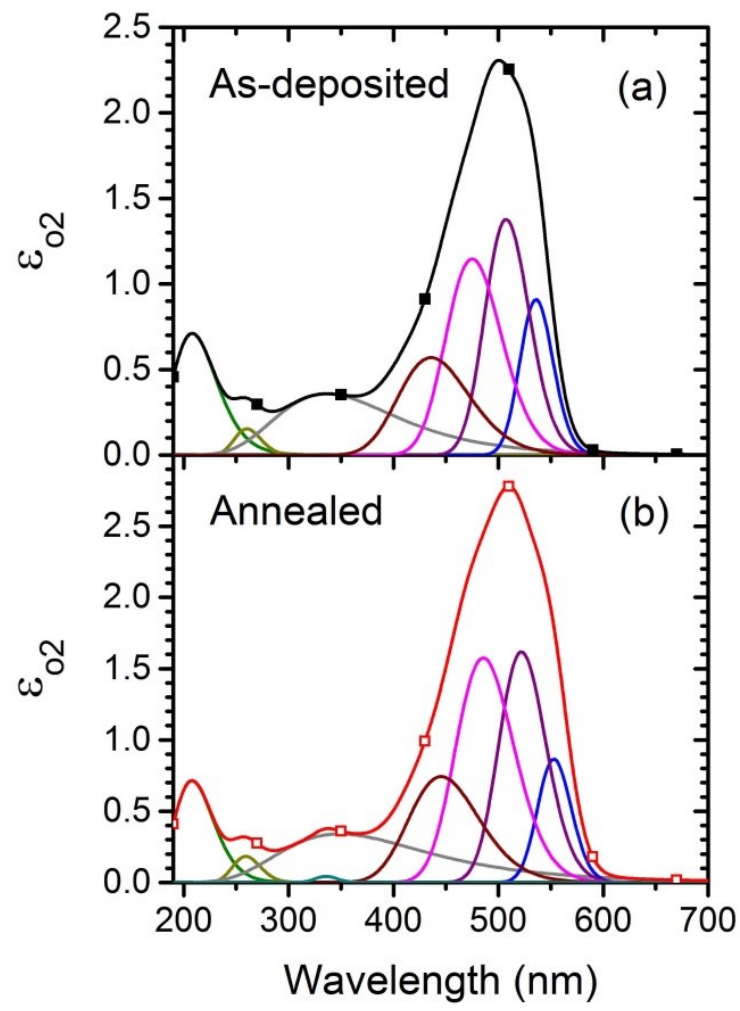


Figure 7

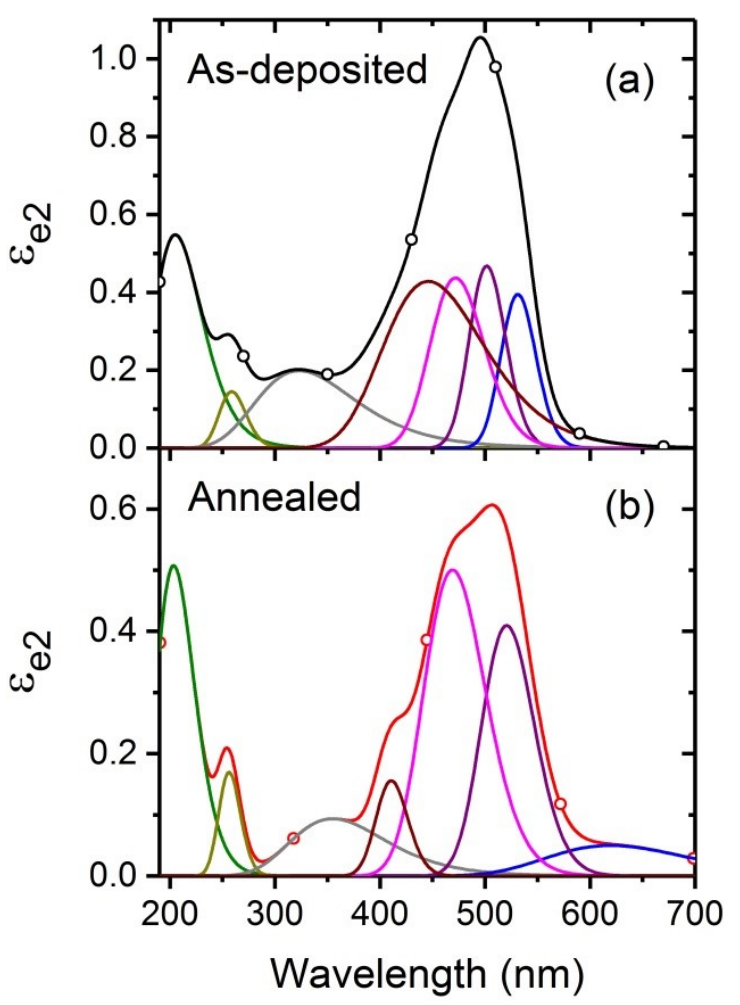


Figure 8

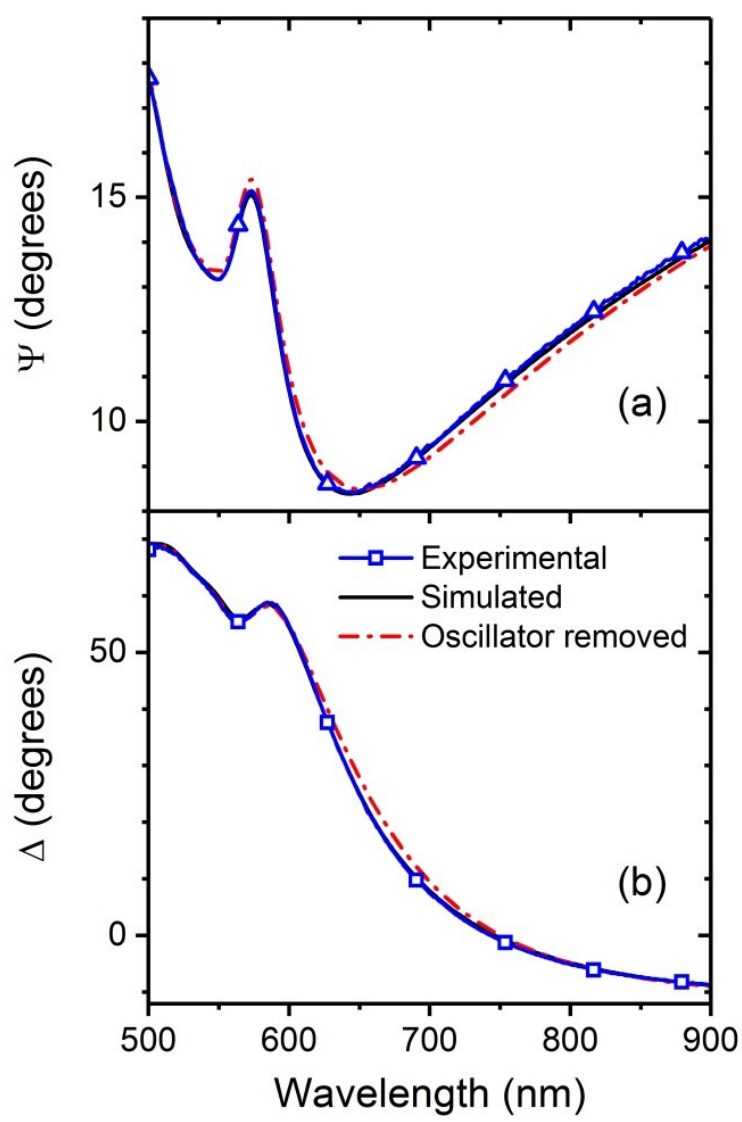


Figure 9
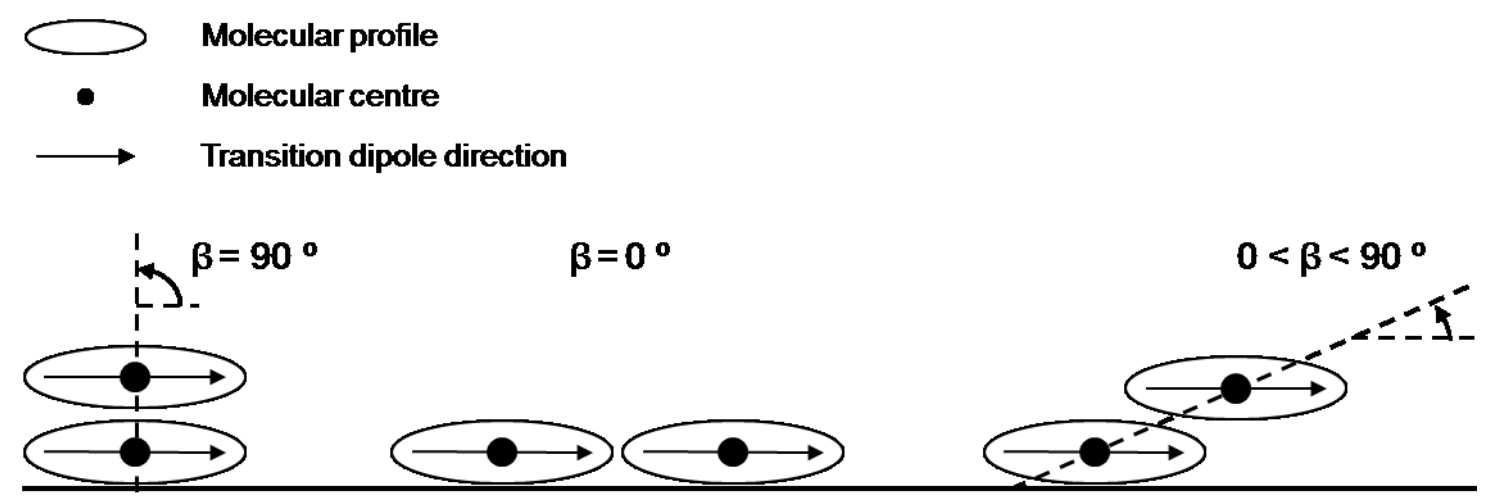

(a) Substrate

(b)

(c)

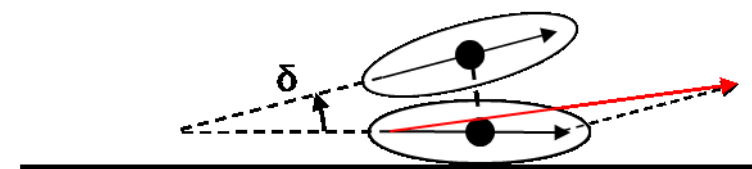

Substrate

(d)

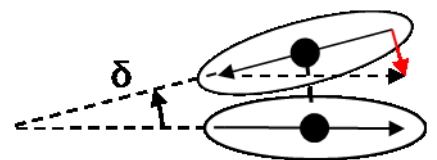

(e) 


\section{References}

[1] J. H. Burroughes, D. D. C. Bradley, A. R. Brown, R. H. Marks, K. Mackay, R. H. Friend, P. L. Burn, and A. B. Holmes, Nature (London) 347, 539 (1990).

[2] A. C. Grimsdale, K. L. Chan, R. E. Martin, P. G. Jokisz, and A. B. Holmes, Chem. Rev. 109, 897 (2009).

[3] M. J. Humphries, R. J. Wilson, O. Fernandez, and R. A. Archer, J. Photon. Energy 1, 011019 (2011).

[4] A. Najari, S. Beaupré, P. Berrouard, Y. Zou, J. -R. Pouliot, C. Lepage-Pérusse, M. Leclerc, Adv. Funct. Mater. 21, 718 (2011).

[5] P. A. Troshin, H. Hoppe, J. Renz, M. Egginger, J. Yu. Mayorova, A. E. Goryachev, A. S. Peregudov, R. N. Lyubovskaya, G. Gobsch, N. S. Sariciftci, and V. F. Razumov, Adv. Funct. Mater. 19, 779 (2009).

[6] C. J. Brabec, S. Gowrisanker, J. J. M. Halls, D. Laird, S. Jia, and S. P. Williams, Adv. Mater. 22, 3839-3856 (2010).

[7] H. Sirringhaus, M. Bird, T. Richards, and N. Zhao, Adv. Mater. 22, 3893-3898 (2010).

[8] P. L. Burn, S.-C. Lo, and I. D. W. Samuel, Adv. Mater. 19, 1675 (2007).

[9] J. P. J. Markham, S.-C. Lo, S. W. Magennis, P. L. Burn, and I. D. W. Samuel, Appl. Phys. Lett. 80, 2645 (2002).

[10] S.-C. Lo, N. A. H. Male, J. P. J. Markham, S. W. Magennis, P. L. Burn, O. V. Salata, and I. D. W. Samuel, Adv. Mater. 14, 975 (2002).

[11] W. L. Leong, G. C. Welch, J. Seifter, J. H. Seo, G. C. Bazan, A. J. Heeger, Adv. Energy Mater. 3, 356 (2013).

[12] C. W. Tang and S. A. VanSlyke, Appl. Phys. Lett. 51, 913 (1987).

[13] T.-W. Lee, T. Noh, H.-W. Shin, O. Kwon, J.-J. Park, B.-K. Choi, M.-S. Kim, D. W. Shin, and Y.-R. Kim, Adv. Funct. Mater. 19, 1625 (2009).

[14] R. Wang, D. Liu, R. Zhang, L. Denga, and J. Li, J. Mater. Chem. 22, 1411 (2012).

[15] M. Cai, T. Xiao, E. Hellerich, Y. Chen, R. Shinar, and J. Shinar, Adv. Mater. 23, 3590 (2011).

[16] J. Chen, C. Shi, Q. Fu, F. Zhao, Y. Hu, Y. Feng, and D. Ma, J. Mater. Chem. 22, 5164 (2012).

[17] I. D. W. Samuel and G. A. Turnbull, Chem. Rev. 107, 1272 (2007).

[18] M. D. McGehee and A. J. Heeger, Adv. Mater. 12, 1655 (2000).

[19] Y. Yang, I. D. W. Samuel, and G. A. Turnbull, Adv. Mater. 21, 3205 (2009).

[20] A. Teichler, J. Perelaer, and U. S. Schubert J. Mater. Chem. C 1, 1910 (2013).

[21] J.-S. Kim, W.-S. Chung, K. Kim, D. Y. Kim, K.-J. Paeng, S. M. Jo, and S.-Y. Jang, Adv. Funct. Mater. 20, 3538 (2010).

[22] R. Saf, M. Goriup, T. Steindl, T. E. Hamedinger, D. Sandholzer, and G. Hayn, Nature Mater. 3, 323 (2004).

[23] T.-Q. Nguyen, I. B. Martini, J. Liu, and B. J. Schwartz, J. Phys. Chem. B 104, 237 (2000).

[24] T.-Q. Nguyen, B. J. Schwartz, R. D. Schaller, J. C. Johnson, L. F. Lee, L. H. Haber, and R. J. Saykally, J. Phys. Chem. B 105, 5153 (2001).

[25] B. J. Schwartz, Annu. Rev. Phys. Chem. 54, 2003 (2003).

[26] S. R. Amrutha and M. Jayakannan, J. Phys. Chem. B 112, 1119 (2008).

[27] C. Y. Yang, F. Hide, M. A. Díaz-García, A. J. Heeger, and Y. Cao, Polymer 39, 2299 (1998).

[28] A. R. Inigo, H.-C. Chiu, W. Fann, Y.-S. Huang, U.-S. Jeng, T.-L. Lin, C.-H. Hsu, K.Y. Peng, and S.-A. Chen, Phys. Rev. B 69, 075201 (2004). 
[29] R. D. Schaller, P. T. Snee, J. C. Johnson, L. F. Lee, K. R. Wilson, L. H. Haber, R. J. Saykally, T.-Q. Nguyen, and B. J. Schwartz, Journal of Chemical Physics 117, 6688 (2002).

[30] G. R. Webster, W. J. Mitchell, P. L. Burn, R. K. Thomas, G. Fragneto, J. P. Markham, and I. D. W. Samuel, J. Appl. Phys. 91, 9066 (2002).

[31] J. C. Ribierre, A. Ruseckas, O. P. M. Gaudin, I. D. W. Samuel, H. Barcena, S. V. Staton, and P. L. Burn, Org. Electron. 10, 803 (2009).

[32] J. M. Winfield, C. L. Donley, and J.-S. Kim, J. Appl. Phys. 102, 063505 (2007).

[33] J. C. Ribierre, S. Watanabe, M. Matsumoto, T. Muto, and T. Aoyama, Appl. Phys. Lett. 96, 083303 (2010).

[34] K. Kanai, T. Miyazaki, H. Suzuki, M. Inaba, Y. Ouchib, and K. Sekib, Phys. Chem. Chem. Phys. 12, 273 (2010).

[35] A. Ruseckas, E.. B. Namdas, M. Theander, M. Svensson, A. Yartsev, D. Zigmantas, M. R. Andersson, O. Inganäs, and V. Sundström, J. Photochem. Photobiol. A 144, 3 (2001).

[36] A. Ruseckas, E.. B. Namdas, T. Ganguly, M. Theander, M. Svensson, M. R. Andersson, O. Inganäs, and V. Sundström, J. Phys. Chem. B 105, 7624 (2001).

[37] J. Liu, T.-F. Guo, and Y. Yang, J. Appl. Phys. 91, 1595 (2002).

[38] T.-W. Lee, O. O. Park, L.-M. Do, and T. Zyung, Synth. Met. 117, 249 (2001).

[39] T.-W. Lee and O. O. Park, Adv. Mater. 12, 801 (2000).

[40] T.-Q. Nguyen, R. C. Kwong, M. E. Thompson, and B. J. Schwartz, Appl. Phys. Lett. 76, 2454 (2000).

[41] Y. Shao, G. C. Bazan, and A. J. Heeger, Adv. Mater. 19, 365 (2007).

[42] I. D. W. Samuel, G. Rumbles, and C. J. Collison, Phys. Rev. B 52, R11573 (1995).

[43] J. W. Blatchford, S. W. Jessen, L.-B. Lin, T. L. Gustafson, D.-K. Fu, H.-L. Wang, T. M. Swager, and A. G. MacDiarmird, Phys. Rev. B 54, 9180 (1996).

[44] G. H. Gelinck, E. G. J. Staring, D.-H. Hwang, G. C. W. Spencer, A. B. Holmes, and J. M. Warmana, Synth. Met. 84, 595 (1997).

[45] U. Lemmer, S. Heun, R. F. Mahrt, U. Scherf, M. Hopmeier, U. Siegner, E. O. Göbel, K. Müllen, and H. Bässler, Chem. Phys. Lett. 240, 373 (1995).

[46] O. P. M. Gaudin, I. D. W. Samuel, S. Amriou, and P. L. Burn, Appl. Phys. Lett. 96, 053305 (2010).

[47] M. Tammer and A. P. Monkman, Adv. Mater. 14, 210 (2002).

[48] M. Campoy-Quiles, P. G. Etchegoin, and D. D. C. Bradley, Phys. Rev. B 72, 045209 (2005).

[49] J. A. Woollam, B. Johs, C. M. Herzinger, J. Hilfiker, R. Synowicki, and C. L. Bungay, in Critical Reviews of Optical Science and Technology, Optical Metrology Vol. CR72, edited by G. A. Al-Jumaily (SPIE, Bellingham, WA, 1999), p. 3.

[50] H. G. Gilch and W. L. Wheelwright, J. Polymer Sci.: A-1 4, 1337 (1966).

[51] S. Son, A. Dodabalapur, A. J. Lovinger, and M. E. Galvin, Science 269, 376 (269).

[52] W. J. Mitchell, C. Pena, and P. L. Burn, J. Mater. Chem. 12, 200 (2002).

[53] W. J. Mitchell, P. L. Burn, R. K. Thomas, G. Fragneto, J. P. Markham, and I. D. W. Samuel, J. Appl. Phys. 95, 2391 (2004).

[54] H. Fujiwara, in Spectroscopic Ellipsometry: Principles and Applications (John Wiley \& Sons Ltd., Chichester, 2007), p. 83.

[55] C. M. Herzinger, B. Johs, W. A. McGahan, J. A. Woollam, and W. Paulson, J. Appl. Phys. 83, 3323 (1998).

[56] B. Johs, R. H. French, F. D. Kalk, W. A. McGahan, and J. A. Woollam, in Optical Interference Coatings; Vol. 2253, edited by F. Abeles (SPIE, 1994), p. 1098.

[57] K. Koynov, A. Bahtiar, T. Ahn, C. Bubeck, and H.-H. Hörhold, Appl. Phys. Lett. 84, 3792 (2004). 
[58] D. McBranch, I. H. Campbell, D. L. Smith, and J. P. Ferraris, Appl. Phys. Lett. 66, 1175 (1995).

[59] T. Tiwald, J.A. Woollam Co., Inc. Annual Newsletter, Iss. 9, p. 4 (Jan. 2008).

[60] D. De Sousa Meneses, M. Malki, and P. Echegut, J. Non-Cryst. Solids 352, 769 (2006).

[61] Yu. N. Gartstein, M. J. Rice, and E. M. Conwell, Synth. Met. 78, 183 (1996).

[62] M. Chandross, S. Mazumdar, S. Jeglinski, X. Wei, Z. V. Vardeny, E. W. Kwock, and E. K. Miller, Phys. Rev. B 50, 14702 (1994).

[63] E. K. Miller, D. Yoshida, C. Y. Yang, and A. J. Heeger, Phys. Rev. B 59, 4661 (1999).

[64] N. Kirova, S. Brazovskii, and A. R. Bishop, Synth. Met. 100, 29 (1999).

[65] M. Chandross, S. Mazumdar, M. Liess, P. A. Lane, Z. V. Vardeny, M. Hamaguchi, and K. Yoshino, Phys. Rev. B 55, 1486 (1997).

[66] C. M. Ramsdale and N. C. Greenham, Adv. Mater. 14, 212 (2002).

[67] G. Lakhwani, R. A. J. Janssen, and S. C. J. Meskers, J. Phys. Chem. B 113, 14165 (2009).

[68] S. Brazovskii, N. Kirova, A. R. Bishop, V. Klimov, D. McBranch, N. N. Barashkov, and J. P. Ferraris, Opt. Mater. 9, 472 (1998).

[69] J. Cornil, D. Beljonne, R. H. Friend, and J. L. Brédas, Chem. Phys. Lett. 223, 82 (1994).

[70] R. D. Schaller, L. F. Lee, J. C. Johnson, L. H. Haber, R. J. Saykally, J. Vieceli, I. Benjamin, T.-Q. Nguyen, and B. J. Schwartz, J. Phys. Chem. B 106, 9496 (2002).

[71] P. W. Bohn, Annu. Rev. Phys. Chem. 44, 37 (1993).

[72] Z. Chen, A. Lohr, C. R. Saha-Möller, and F. Würthner, Chem. Soc. Rev. 38, 564 (2009).

[73] H. Yao, K. Domoto, T. Isohashi, and K. Kimura, Langmuir 21, 1067 (2005).

[74] A. P. Marchetti, C. D. Salzberg, and E. I. P. Walker, J. Chem. Phys. 64, 4693 (1976).

[75] E. Peeters, A. M. Ramos, S. C. J. Meskers, and R. A. J. Janssen, J. Chem. Phys. 112, 9445 (2000).

[76] S. C. J. Meskers, R. A. J. Janssen, J. E. M. Haverkort, and J. H. Wolter, Chem. Phys. 260, 415 (2000).

[77] S.-H. Lim, T. G. Bjorklund, and C. J. Bardeen, J. Phys. Chem. B 108, 4289 (2004).

[78] G. A. Sherwood, R. Cheng, T. M. Smith, J. H. Werner, A. P. Shreve, L. A. Peteanu, and J. Wildeman, J. Phys. Chem. C 113, 18851 (2009).

[79] S. Blumstengel, F. Meinardi, P. Spearman, A. Borghesi, R. Tubino, and G. Chirico, J. Chem. Phys. 117, 4517 (2002).

[80] J. Gierschner, H.-J. Egelhaaf, D. Oelkrug, and K. Müllen, J. Fluor. 8, 37 (1998).

[81] S. Siddiqui and F. C. Spano, Chem. Phys. Lett. 308, 99 (1999).

[82] J. Kim, Pure Appl. Chem. 74, 2021 (2002).

[83] J. Kim and T. M. Swager, Nature 411, 1030 (2001).

[84] Y. F. Huang, Y. J. Shiu, J. H. Hsu, S. H. Lin, A. C. Su, K. Y. Peng, S. A. Chen, and W. S. Fann, J. Phys. Chem. C 111, 5533 (2007).

[85] X.-T. Hao, N. Y. Chan, D. E. Dunstan, and T. A. Smith, J. Phys. Chem. C 113, 11657 (2009).

[86] F. C. Spano, J. Chem. Phys. 122, 234701 (2005).

[87] E. T. Niles, J. D. Roehling, H. Yamagata, A. J. Wise, F. C. Spano, A. J. Moulé, and J. K. Grey, J. Phys. Chem. Lett. 3, 259 (2012).

[88] H. Yamagata and F. C. Spano, J. Chem. Phys. 136, 184901 (2012).

[89] H. Yamagata, N. J. Hestand, F. C. Spano, A. Köhler, C. Scharsich, S. T. Hoffmann, and H. Bässler, J. Chem. Phys. 139, 114903 (2013). 
[90] M. Kasha, H. R. Rawls, and M. Ashraf El-Bayoumi, Pure Appl. Chem. 11, 371 (1965).

[91] R. Chakraborty and L. Rothberg, J. Phys. Chem. A 120, 551 (2016). 